\title{
PERSPECTIVAS DEL USO DE LA SEMILLA (SEXUAL) DE PAPA EN NICARAGUA
}

\author{
Fidel Torres; C. González; H. Torres; L. Aguilar; P. Blandón
}

RESUMEN

El Programa Nacional de Papa en Nicaragua investiga la posibilidad de empleo de la semilla sexual en la producción de tubérculo-semilla y/o papa para consumo. Los trabajos realizados en las zonas productoras de papa, ubicadas entre los 900 y 1330 msnm, están orientados hacia la producción de semilla híbrida y de polinización libre (PL) a partir de progenitores selectos. También a la evaluación y selección de progenies promisorias para la producción de tubérculos en almácigos, utilizables luego como tubérculossemillas en campos comerciales. En las zonas de mayor elevación y en los meses de marzo a junio se observaron las mejores condiciones para la producción de semilla. Los cruzamientos logrados con mayor facilidad fueron aquellos de Atzimba y Serrana-INTA (femeninos) con R128.6 y 7XY.1 (masculinos), con rendimientos de 1.07 a $0.74 \mathrm{~g} /$ planta. En la producción de semilla de PL, los clones DTO-28, DTO-33 y 7XY.1 tuvieron rendimientos de $0.40,0.54$ y $1.07 \mathrm{~g}$ de semilla por planta. Las progenies producidas por cruzamientos entre Atzimba y Serrana INTA con R128.6, 7XY.1, DTO-28 y DTO-33, evaluadas a $950 \mathrm{msnm}$ tuvieron rendimiento similar de tubérculos (7.2 a $8.8 \mathrm{~kg} / \mathrm{m}^{2}$ ); en cambio a $1330 \mathrm{msnm}$, las progenies Atzimba x R128.6, Atzimba x 7XY.1 y Atzimba x DTO-28 expresaron mejor su potencial de rendimiento alcanzado $11.8,10.4$ y $9.4 \mathrm{~kg} / \mathrm{m}^{2}$, respectivamente. Las cuatro progenies de PL locales, 7XY.1, DTO-28, DTO-33 y Desirée, rindieron 8.1, 6.4, 5.7 y $6.0 \mathrm{~kg} / \mathrm{m}^{2}$, respectivamente.

Palabras Claves Adicionales: TPS, vigor de semilla, polinización libre, Producción de semilla, progenitores, semilla, rendimiento.

Aceptado para publicación: Mayo 16, 1991

* Investigadores. Programa Nacional de Papa. Ministerio de Agricultura y Ganadería (MAG) Región I, "Las Segovias". Estelf. Nicaragua. 


\section{SUMMARY}

\section{PERSPECTIVES OF THE USE OF TRUE POTATO SEED IN NICARAGUA}

The Nicaraguan National Potato Program is investigating the possibility of the use of true potato seed in the production of tuber seed and/or ware potatoes for direct consumption. Investigations undertaken in the potato producing regions, situated at between 900 and 1330 masl, are oriented towards the production of hybrid and open pollinated (OP) seed from selected progenitors. Evaluation and selection of promising progenies for an intensive tuber production in seed beds for tuber seed production, is also investigated. The best conditions for the production of true potato seed were obtained at the higher elevations areas and from March to June. The best results were obtained when Atzimba and Serrana-INTA (females) were crossed with R128.6 and 7XY.1 (males) with yields between 1.07 to $0.74 \mathrm{~g} /$ plant. In the production of OP seed the clones DTO-28, DTO-33 and 7XY.1 yielded 0.40, 0.54 and $1.07 \mathrm{~g}$ of seed per plant, respectively. The true seed obtained from crosses of Serrana-INTA and Atzimba with DTO-28, DTO-33, 7XY and R128.6, evaluated at 950 masl, had similar tuber yield $\left(7.2\right.$ to $\left.8.8 \mathrm{~kg} / \mathrm{m}^{2}\right)$ while at 1330 masl the progenies Atzimba x R128.6, Atzimba x 7XY.1 and Atzimba x DTO-28 better expressed their yield potential reaching 11.8, 10.4 and $9.4 \mathrm{~kg} / \mathrm{m}^{2}$, respectively. The local OP progenies from 7XY.1, DTO-28, DTO-33 and Desirée yielded 8.1, 6.4, 5.7 and $6.0 \mathrm{~kg} / \mathrm{m}^{2}$, respectively.

Additional Index Words: TPS, seed vigor, open pollination, seed production, progenitors, yield.

El Programa Nacional de Papa (PNP) de Nicaragua está investigando la posibilidad del empleo de la semilla sexual (en adelante sólo semilla) como sistema complementario al tradicional en la producción de papa, por constituir una alternativa económica para la producción de tubérculos sanos $(1,9,16,17,20)$. Esta alternativa es especialmente más viable para pequeños productores que no tienen acceso al sistema de distribución de tubérculossemillas del PNP y que, en consecuencia, no son desalentados 
por las desventajas que pueda tener su uso. Los esfuerzos iniciales se realizan en dos direcciones: evaluar y seleccionar progenies de semilla híbrida y de polinización libre (PL) para producción de tubérculos-semillas en camas de crecimiento; además, identificar ambientes favorables para la producción de semilla.

En el análisis de la factibilidad de esta tecnología, es importante evaluar el potencial de la semilla a utilizar, bajo las condiciones particulares de las zonas de producción. Las progenies híbridas producidas por programas de semilla especializados son generalmente superiores a las de polinización libre (PL) en vigor en la emergencia, desarrollo inicial de plántulas y rendimiento de tubérculos. Hay excepciones cuando los progenitores para semilla de PL son debidamente seleccionados $(3,10)$.

El éxito del uso de la semilla en la producción comercial de papa depende básicamente de la calidad y cantidad de semilla que se disponga (19). En el caso de que la semilla se deba producir localmente, se deben seguir los siguientes pasos:

- Elección de progenitores de buenas características de rendimiento y de tubérculo, resistencia a enfermedades y de producción de semilla;

- $\quad$ identificación de ambientes adecuados que favorezcan la producción de semilla, y

- manejo agronómico apropiado de las plantas madres durante el desarrollo de la semilla para optimizar su calidad $(13,15)$.

El presente trabajo muestra los resultados en la producción de semilla híbrida y de PL a partir de progenitores selectos en tres ambientes diferentes y dos épocas distintas del año. Se muestra también la evaluación del vigor y rendimiento de tubérculos de diferentes progenies de semilla híbrida y de PL provenientes de clones selectos y de variedades europeas adaptadas.

\section{MATERIALES Y METODOS}

\section{Producción de Semilla}

Los experimentos se llevaron a cabo en tres altitudes diferentes y en dos períodos distintos del año. En el primer período (marzo a junio de 1988), 
los trabajos se condujeron en la Estación Experimental de "San José" ubicada a 1330 msnm (temp. prom. min. $15^{\circ} \mathrm{C} /$ máx. $21^{\circ} \mathrm{C}$ ) y en el Centro Experimental de Estelí (Tem. prom. min. $21^{\circ} \mathrm{C} /$ máx. $27^{\circ} \mathrm{C}$ ); la longitud promedio del día, en este período fue de 13:30 hrs. En el segundo período (octubre 88 a enero 89) los experimentos se realizaron en la finca "El Diamante" (Tem. prom. min. $16^{\circ} \mathrm{C} /$ máx. $24^{\circ} \mathrm{C}$ ); la longitud promedio del día fue de 12 hrs.

Tubérculos de las variedades Atzimba y Serrana-INTA, usadas como hembras, y los clones R128.6, 7XY.1, DTO-28 y DTO-33, usados como machos, fueron proporcionados por el Centro Internacional de la Papa (CIP) en agosto de 1987. Las plantaciones se hicieron para el primer y segundo períodos el 25 de marzo y el 1 de octubre, respectivamente. La profundidad de plantación fue de $5.0 \mathrm{~cm}$. Las plantas no se aporcaron, las ramas laterales se cortaron y posteriormente las plantas se sostuvieron con cuerdas apoyadas en estacas. A partir de la aparición de los botones florales, en los machos y hembras, se hicieron seis aplicaciones suplementarias de nitrógeno $(\mathrm{N})$ cada diez días, a razón de $5.0 \mathrm{~g}$ de N/planta. En los progenitores masculinos el polen se colectó de las primeras inflorescencias, almacenándolo en cápsulas de gelatina dentro de frascos conteniendo arroz tostado como desecante y herméticamente cerradas. En el primer período, debido al escaso número de plantas, el polen fue colectado de las primeras inflorescencias; las inflorescencias posteriores de estas mismas plantas se dejaron para la producción de semilla de polinización libre (PL). En el segundo período se destinaron plantas por separado para cada fin. La emasculación se hizo con un día de anticipación a la polinización, la cual se realizó entre las 8 y las 10 de la mañana, repitiendo la operación por tres días consecutivos.

En el primer período los machos se plantaron quince días antes que las hembras. El polen fue almacenado a temperatura ambiente. Las bayas se cosecharon ocho semanas después de la polinización cuando las plantas estaban completamente maduras.

En el segundo período, los machos se plantaron con un mes de anticipación que las hembras y se adicionó la variedad Desirée, por su adaptación a estas regiones y su buena capacidad de fructificación. El polen fue almacenado a $5^{\circ} \mathrm{C}$ y transportado al campo en una caja térmica con hielo. Las bayas se cosecharon siete semanas después de la polinización, cuando las plantas estuvieron completamente maduras.

La extracción de la semilla se hizo manualmente, se secó a temperatura ambiente $\left(20^{\circ} \mathrm{C}\right)$ y se almacenó en recipientes conteniendo arroz tostado y herméticamente cerrados, asegurando baja humedad relativa. El peso de 100 semillas se consiguió pesando tres muestras de cada progenie, empleando una balanza analítica. 


\section{Vigor y Rendimiento de Tubérculos de Progenies de Semilla.}

Las camas utilizadas fueron de $1.4 \mathrm{~m}$ de ancho y $0.2 \mathrm{~m}$ de alto, utilizando como substrato suelo franco, desinfestado con bromuro de metilo. Poco antes de la siembra, se incorporó 200 g de la formulación 10-30-10 (N-P-K), por m². A partir de la segunda semana, después de la emergencia, se hicieron seis fertilizaciones adicionales, una por semana, aplicadas en dilución con regadera haciendo un total de $60 \mathrm{~g}$ de $\mathrm{N}$ por $\mathrm{m}^{2}$, durante todo el periodo vegetativo. La densidad de siembra fue de 100 plantas por $\mathrm{m}^{2}$, colocándose $3 \mathrm{a}$ 4 semillas por punto de siembra y a $0.5 \mathrm{~cm}$ de profundidad. A los 28 días después de la siembra, cuando las plantas alcanzaron un promedio de $5.0 \mathrm{~cm}$ de altura, se procedió al raleo, eliminando las más débiles para dejar finalmente 100 plántulas por $\mathrm{m}^{2}$. Dos días después del raleo se realizó el primer aporque y 10 días después el segundo, colocándose una capa de suelo franco desinfestado de $3.0 \mathrm{~cm}$ en cada operación. Las plantas permanecieron en las camas hasta la cosecha a los 100 días después de la siembra.

1. Evaluación del vigor y rendimiento de tubérculos de progenies de semilla híbrida y PL de variedades importadas adaptadas.

Este experimento se llevó a cabo en la Estación Experimental "San José" de enero a abril de 1988. Las progenies evaluadas: Atzimba x DTO-28 y Serrana x DTO-28 fueron proporcionadas por el CIP y las de PL de las variedades Desirée y Baraka fueron obtenidas en Nicaragua en campos de producción comercial. El diseño experimental fue de bloques completos al azar con cuatro repeticiones. Cada unidad experimental fue de $1.5 \mathrm{~m}^{2}$ de área de cama. El vigor de las progenies se evaluó considerando valores por $\mathrm{m}^{2}$ de altura de plántula, porcentaje de sobrevivencia y cobertura de suelo, evaluados a los 28 días después de la siembra. El rendimiento (peso tubérculos por $\mathrm{m}^{2}$ ) se clasificó en cinco rangos: < 5, 5 a 10, 10 a 20, 20 a 40 y $>40$ gramos. Se consideró que los tubérculos mayores de $5 \mathrm{~g}$ son utilizables como tubérculosemilla convencional.

2. Evaluación del rendimiento de tubérculos de progenies de semilla híbrida en dos altitudes diferentes.

Este experimento se realizó simultáneamente en la Estación Experimental "San José" (1330 msnm) y en la finca “el Diamante” (950 msnm) de septiembre a diciembre de 1988. Las progenies provenientes del CIP fueron 
tratadas con ácido giberélico (AG) a 1500 ppm. Se usó el diseño de bloques completos al azar con cuatro repeticiones; cada unidad experimental fue de 1.5 $\mathrm{m}^{2}$.

3. Evaluación del vigor de progenies de semilla híbrida y PL producidas en Nicaragua.

Este experimento se realizó en la finca "El Diamante" (950 msnm) de septiembre a diciembre de 1988. Las progenies evaluadas fueron: Serrana $\mathrm{x}$ R128.6, Serrana x 7XY.1, Atzimba x R128.6 y Atzimba x 7XY.1 y de PL: R128.6, 7XY.1, DTO-28 y DTO-33, producidas entre los meses de marzo a junio de 1988 en la Estación Experimental "San José", Jinotega. También se incluyeron semillas de PL de Desirée obtenida de campos de producción de tubérculos-semillas. Las semillas fueron tratadas con AG a 1500 ppm. El vigor fue medido por la velocidad de emergencia (CoV), calculado por conteos diarios de emergencia de plántulas, a partir de su inicio y durante 15 días. El porcentaje de sobrevivencia se midió a los 28 días después de la siembra. El $\mathrm{CoV}$ se calculó mediante la fórmula: $\mathrm{CoV}=100(\mathrm{Ni} / \mathrm{NiTi})$ donde $\mathrm{Ni}=$ número de plántulas emergidas en el día i, y Ti = días después de la siembra. Sólo en las progenies de PL se continuó la evaluación hasta rendimiento de tubérculos por $\mathrm{m}^{2}$ de cama, datos que no pudieron ser tomados en las progenies híbridas a causa de daño mecánico en varias de sus parcelas. El diseño experimental fue de bloques completos al azar con cuatro repeticiones; cada unidad experimental fue de $1.5 \mathrm{~m}^{2}$.

\section{RESULTADOS Y DISCUSIÓN}

\section{Producción de Semilla}

Las elevadas temperaturas predominantes (mín. $21^{\circ} \mathrm{C} /$ máx. $27^{\circ} \mathrm{C}$ ) durante el primer período (marzo-jumo) en la zona baja del Valle de Estelí, (800 msnm), donde se ubica el Centro Experimental de Estelí posiblemente afectaron la viabilidad de los gametos, tanto de los progenitores masculinos como de los femeninos. Fracasaron todas las hibridaciones y hubo ausencia de autopolinizaciones en las inflorescencias que se destinaron para este propósito. Las temperaturas moderadas de las zonas elevadas (prom. mín. $15^{\circ} \mathrm{C} /$ máx. $21^{\circ} \mathrm{C}$ ) como en la Estación Experimental de "San José" durante este período, no afectaron la fertilidad de los parentales, por lo que se logró producir semilla híbrida y de PL. Además de las temperaturas favorables, la 
mayor longitud del día en esta época del año (13.5hr) posiblemente favoreció una mejor floración y fructificación. La floración de DTO-28 y DTO-33 se limitó sólo a una o dos inflorescencias y la producción de polen fue muy escasa. DTO-33 no conservó su fertilidad y el polen DTO-28 perdió rápidamente su viabilidad (a los 15 o 20 días de su colección). Con 7XY.1 y R128.6 sin embargo, sucedió lo contrario.

Tuvieron una profusa y constante floración produciendo abundante polen cuya fertilidad se conservó después de 30 días de almacenamiento a temperatura ambiente. Estas diferencias de producción y conservación de fertilidad de polen en los progenitores masculinos se expresaron en el logro diferenciado de producción de semilla híbrida. Los rendimientos más bajos se obtuvieron en los cruzamientos con DTO-28, mientras que en los cruzamientos con 7XY.1 y R128.6 fueron mucho mayores (Tabla 1). Los rendimientos de semilla híbrida y de PL obtenidos corresponden a resultados similares reportados por Pallais (15) bajo condiciones de trópicos cálidos similares a Nicaragua (Tabla 1). Estos rendimientos son bajos en relación a lo que se puede obtener en condiciones más favorables para producir semilla. Será necesario por consiguiente hacer más investigación para optimizar su producción en ambientes tropicales.

En el segundo período, se obtuvieron resultados similares al período anterior en el tipo de semilla producida y mejor lograda. Sin embargo, los rendimientos de semilla por planta fueron muy inferiores (Tabla 1). Durante el segundo período parece haber actuado desfavorablemente: la temperatura relativamente alta (prom. mín. $16^{\circ} \mathrm{C} /$ máx $24^{\circ} \mathrm{C}$ ) a esta altitud (950 msnm), y la longitud del día, que en estos meses son los más cortos del año (12 hr). El efecto de la longitud del día se pudo observar en los machos, especialmente en DTO-28 y DTO-33, sembrados un mes antes que las hembras. Se observó inhibición de la floración o caída de botones florales, que fue completa en DTO-33 y hasta del 50\% en el caso de DTO-28. Como consecuencia hubo una pobre formación de bayas de PL (sólo 3 a 4) por planta, produciendo 0.1 g de semilla/planta. Las progenies de PL 7XY.1 y R128.6 fueron, sin embargo, menos afectadas, produciendo 0.3 y $1.05 \mathrm{~g}$ semilla/planta, respectivamente. En las hembras Atzimba y Serrana, que iniciaron su floración a mediados de diciembre, no se observó declinación apreciable en su floración; sus cruzamientos con DTO-28, 7XY.1 y R128.6 tuvieron bajos rendimientos de semilla (Tabla 1). El cultivar Desirée, que comúnmente fructifica abundantemente en los meses de mayo a septiembre, su floración fue completamente inhibida, tanto en el área experimental como en los campos comerciales, observación que se confirma todos los años en esta época. Estos resultados confirman lo dificultoso que puede ser producir semilla híbrida en los trópicos como Nicaragua.

El hecho de que la semilla de los cruzamientos con Serrana -en el primer periodo- obtuvo mayor peso (peso de 100 semillas) que la de los 
cruzamientos con Atzimba, está posiblemente asociado a que los híbridos de Serrana tuvieron menos semilla por baya (14).

Tabla 1. Producción de semilla híbrida y de polinización libre (PL) en tres ambientes diferentes y en dos épocas del año expresada en gramos de semilla por planta.

\begin{tabular}{lccc}
\hline & “San José” & Estelí & "El Diamante" \\
PROGENIE & $\begin{array}{c}\text { (1330 msnm) } \\
\text { Marzo -Junio }\end{array}$ & $\begin{array}{c}(800 \mathrm{msnm}) \\
\text { Marzo-Junio'88 }\end{array}$ & $\begin{array}{c}(900 \mathrm{msnm}) \\
\text { Oct.-Enero'89 }\end{array}$ \\
\hline & & & \\
HÍBRIDOS & & & \\
Serrana x DTO-2S & 0.19 & 0.0 & 0.10 \\
Serrana x 7XY.1 & 1.07 & 0.0 & 0.34 \\
Serrana x R128.6 & 0.74 & 0.0 & 0.32 \\
Atzímba x DTO.28 & 0.03 & 0.0 & 0.05 \\
Atzimba x 7XY.1 & 0.83 & 0.0 & 0.53 \\
Alzimba x R128.6 & 0.97 & 0.0 & 0.38 \\
POLINIZACION LIBRE & & & \\
DTO-28 & 0.40 & 0.0 & 0.10 \\
DTO-33 & 054 & 0.0 & 0.00 \\
7XY.1 & 1.29 & 0.0 & 0.30 \\
R128.6 & 7.72 & 00 & -- \\
\hline
\end{tabular}

En el segundo período, sin embargo, la semilla híbrida de Atzimba aumentó significativamente su peso, superando ligeramente a los híbridos de Serrana que mantuvieron pesos similares en ambos periodos (Tabla 2). Las progenies DTO-28 y 7XY.1, produjeron la semilla de mayor peso en ambos periodos. La R128.6 aumentó significativamente el peso de su semilla en el segundo periodo, debido posiblemente a que su floración no fue tan profusa como en el primer periodo, con la consecuente menor cantidad de bayas, lo que debe haber influenciado en el mayor peso de la semilla (2) (Tabla 2).

A pesar que los cruzamientos con DTO-28 (en ambas localidades y periodos) produjeron semillas con el mayor peso (Tabla 2), buen vigor y rendimiento aceptable de tubérculos (Tablas 3,4 y 5). Estos sin embargo produjeron baja cantidad de semillas por planta (Tabla 1) debido a las escasas fecundaciones logradas con su polen. Algo similar ocurre con DTO-33. Por otro lado 7XY.1 y R128.6, produjeron semilla híbrida en cantidades significativamente mayores debido a su gran capacidad de floración y producción de polen. Para las condiciones de la Estación Experimental "San José", DTO-33, DTO-28 y 
7XY.1, han mostrado ser eficaces por su buena producción de bayas de PL, semillas de buen vigor y rendimiento de tubérculos aceptable. R128.6, que tiene una excesiva fructificación, genera semilla liviana, de muy bajo vigor y pobre rendimiento de tubérculos (Tablas 2 y 6). La variedad Desirée, adaptada a estas regiones, también mostró buen comportamiento para la producción intensiva de semilla de PL de buen vigor. La producción de semilla de Desirée puede mejorarse con un mejor manejo agronómico de las plantas. Desirée tiene una alta capacidad de floración y fructificación, particularmente durante el periodo marzo a agosto.

Tabla 2. Peso de 100 semillas (mg) de progenies híbridas y de polinización libre (PL) producidas en dos ambientes diferentes; Estación Experimental "San José" y la finca "El Diamante".

\begin{tabular}{lcc}
\hline PROGENIE & $\begin{array}{c}\text { "San José” } \\
\text { (1330 masnm) } \\
\text { Marzo-Junio'88 }\end{array}$ & $\begin{array}{c}\text { "El Diamante" } \\
\text { (900 msnm) } \\
\text { Oct'88-Ene'89 }\end{array}$ \\
HÍBRIDOS & 77.8 & 78.6 \\
Serrana x DTO-28 & 72.2 & 71.2 \\
Serrana x 7XY.1 & 70.0 & 66.0 \\
Serrana X R128.6 & 66.4 & 75.1 \\
Alzimba x 7XY.1 & 60.1 & 74.0 \\
Atzimba x R128.6 & 3.5 & 65 \\
Tuckey (p = 0.05) & & \\
& & \\
POLINIZACION LIBRE & & 70.6 \\
DTO-28 & 70.9 & 68.1 \\
7XY.1 & 68.6 & - \\
DTO-33 & 60.0 & 67.6 \\
R128.6 & 57.9 & 5.0 \\
Tuckey (p = 0.05) & 9,4 & \\
\hline
\end{tabular}

En general, los rendimientos de semilla logrados en estas pruebas iniciales han sido superiores o corresponden, según el caso, a lo esperado para las regiones tropicales de baja altitud, en las que según Pallais (15), los rendimientos de semilla están por debajo de los 0.5 g/planta. Además, el peso de semilla (peso de 100 semillas) de las diferentes progenies híbridas y de PL producidas son aceptables. Sin embargo, las cantidades de semilla híbrida producida son muy inferiores en comparación a lo que se obtiene en condiciones favorables (15), lo que elevaría su costo de producción. De otro lado, la semilla PL puede constituirse en una posibilidad de producción local de semilla a continuar investigando especialmente en la determinación de la mejor época de producción en las zonas ubicadas entre los 1300 a 1400 msnm. 


\section{Vigor y Rendimiento de Tubérculos de Progenies de Semilla}

Resultados satisfactorios fueron obtenidos en la producción de tubérculos de primera generación para su posterior empleo como tubérculos-semillas. El substrato usado fue únicamente suelo-franco, en lugar de costosas mezclas de suelo con materia orgánica (musgo) y/o arena $(1,7,11,21)$. Se ha empleado también una fertilización de NPK de fácil adquisición y bajo costo en el mercado. Los rendimientos de tubérculos de 8.0 a $11.8 \mathrm{~kg} / \mathrm{m}^{2}$, bajo estas condiciones de manejo, iguala o supera otras experiencias realizadas con substratos de mezclas y fuentes de nutrientes $(4,9,23)$.

1. Vigor y rendimiento de tubérculos de progenies de semilla híbrida y de PL de variedades importadas adaptadas.

Las progenies Atzimba x DTO-28 y Serrana x DTO-28 mostraron ser superiores en rendimiento de tubérculos por $\mathrm{m}^{2}\left(9.2\right.$ y $\left.8.6 \mathrm{~kg} / \mathrm{m}^{2}\right)$ a las progenies PL de Desirée y Baraka (6.5 y $\left.6.1 \mathrm{~kg} / \mathrm{m}^{2}\right)$ (Tabla 3). Sin embargo, en lo referente al vigor inicial de plántulas, de acuerdo a los parámetros empleados, los híbridos fueron superiores sólo a Baraka (PL), más no a Desirée (PL) (Tabla 4). Atzimba x DTO-28 produjo el mayor número de tubérculos superiores a $5 \mathrm{~g} / \mathrm{m}^{2}$. En cambio, fue Serrana x DTO-28 la que tuvo mayor porcentaje de tubérculos mayores de $5 \mathrm{~g} / \mathrm{m}^{2}$ (83\%) (Tabla 4). Las progenies de PL tuvieron un rendimiento aceptable de tubérculos mayores de 5 g por $\mathrm{m}^{2}$ (>60\%), lo que significa una mayor posibilidad de recuperación de tubérculos utilizables como "semilla" por unidad de superficie sembrada. Además, Desirée (PL) dio plántulas de tan buen vigor como la de los híbridos pese a que su semilla se desarrolló bajo condiciones subóptimas y que las bayas fueron cosechadas antes de su maduración completa. Estos resultados muestran que Serrana x DTO-28 y Atzimba x DTO-28 son progenies promisorias. Además de la posibilidad de emplear Desirée (PL) como una opción en la producción intensiva de semilla de bajo costo; la cual puede ser producida apropiadamente por el propio productor.

2. Rendimiento de tubérculos de progenies híbridas en dos ambientes diferentes en altitud.

Los resultados de estos experimentos mostraron que algunas progenies híbridas como Atzimba x R128.6 (11.8 kg/m²), Atzimba x 7XY:1 (10.4 $\left.\mathrm{kg} / \mathrm{m}^{2}\right)$ y Atzimba x DTO-28 $\left(9.4 \mathrm{~kg} / \mathrm{m}^{2}\right)$ expresaron mejor su potencial de rendimiento de tubérculos en el ambiente de mayor altitud (1330 msnm). 
Los rendimientos (Tabla 5) pueden considerarse aceptables comparados con los obtenidos por Wiersema (23) y en el CIP $(4,5)$. En el ambiente de menor altitud (950 msnm), el conjunto de las progenies evaluadas tuvieron rendimientos similares entre ellas, los cuales variaron entre 7.2 y $8.8 \mathrm{~kg} / \mathrm{m}^{2}$ (Tabla 5).

3. Vigor de progenies de semilla híbridas y de PL producidas en Nicaragua.

De las progenies híbridas producidas localmente, las de PL mostraron buena velocidad de emergencia (CoV) en relación a los híbridos, especialmente 7XY.1 (Tabla 6), aunque los valores alcanzados, inferiores a 20, son considerados como bajos según la escala propuesta por CIP (6). Los porcentajes de germinación, superiores al 80\%, son aceptables para ambos tipos de progenie (Tabla 6). Además en la mitad de las progenies evaluadas el porcentaje de sobrevivencia fue superior al $90 \%$. De acuerdo a los parámetros de vigor escogidos, los valores obtenidos por las progenies híbridas no fueron significativamente diferentes a las de PL, con el rendimiento de tubérculos, sin embargo, los resultados pueden variar, por ser dos momentos fisiológicamente diferentes y relativamente independientes en la expresión del genotipo (15).

Para las condiciones en que fueron llevados estos estudios, el rendimiento de tubérculos de las progenies de PL pueden considerarse como buenos (Tabla 6) y en particular el del 7XY.1 y DTO-28 $\left(8.1\right.$ y $\left.6.4 \mathrm{~kg} / \mathrm{m}^{2}\right)$ que igualan al de algunas híbridos. Esto es importante porque progenies de PL son de fácil obtención y de bajo costo (10) lo que las convierte en una alternativa atractiva para la producción intensiva.

Las condiciones ambientales de las zonas de producción de papa de Nicaragua ubicadas por encima de $800 \mathrm{msnm}$ ofrecen buenas posibilidades para la producción de papa a partir de semilla, especialmente durante la época lluviosa que permite suministrar la humedad suficiente y prescindir de sombras protectoras.

Es de gran importancia lograr que el manejo de la semilla se asemeje a la modalidad que el productor emplea para otras hortalizas cuyas semillas tienen un vigor inherente superior al de la papa. 
Tabla 3. Vigor de plántulas provenientes de semilla híbrida y de polinización libre (PL) evaluados a los 28 días después de la siembra en la Estación Experimental "San José" (1330 msnm).

\begin{tabular}{lccc}
\hline \multicolumn{1}{c}{ PROGENIE } & $\begin{array}{c}\text { ALTURA } \\
\text { PLANTA } \\
(\mathrm{cm})\end{array}$ & $\begin{array}{c}\text { SOBREV. } \\
(\mathbf{\%})\end{array}$ & $\begin{array}{c}\text { COBERTURA } \\
\text { SUELO/m }\end{array}$ \\
& & & \\
& & & \\
Atzimba x DTO.28 & 3.9 & 82.0 & 55.0 \\
Serrana x DTO.28 & 4.9 & 77.0 & 72.5 \\
Desireé (PL) & 3.7 & 83.5 & 67.5 \\
Baraka (PL) & 2.9 & 69.5 & 25.0 \\
\hline Duncan (p= 0.05) & $\mathbf{0 . 4 5}$ & $\mathbf{5 . 4}$ & $\mathbf{2 0 . 5}$ \\
\hline
\end{tabular}

Tabla 4. Rendimiento por $\mathrm{m}^{2}$ en camas de progenies de semilla híbrida y de polinización libre (PL) en la Estación Experimental "San José" (1300 msnm).

\begin{tabular}{lcccc}
\hline \multicolumn{1}{c}{ PROGENIE } & $\begin{array}{c}\text { REND. } \\
(\mathrm{kg} / \mathrm{m} 2)\end{array}$ & $\begin{array}{c}\text { No. TOTAL } \\
\text { Tub./m² }\end{array}$ & $\begin{array}{c}\text { No. TUB. } \\
>5 \mathrm{~g}\end{array}$ & $\begin{array}{c}\text { \%TUB } \\
>5 \mathrm{~g}\end{array}$ \\
\hline Atzimba x DTO.28 & 9.2 & 581 & 473 & 81.4 \\
Serrana X DTO.28 & 8.6 & 365 & 305 & 83.6 \\
Desiré (PL) & 6.5 & 447 & 341 & 76.3 \\
Baraka (PL) & 6.1 & 433 & 272 & 62.8 \\
\hline Duncan (p=0.05) & $\mathbf{1 . 5}$ & $\mathbf{1 0 0}$ & $\mathbf{8 0}$ & $\mathbf{6 . 2}$ \\
\hline
\end{tabular}

Tabla 5. Rendimiento $\left(\mathrm{kg} / \mathrm{m}^{2}\right)$ de progenies de semilla híbrida obtenidas del CIP en dos altitudes de Nicaragua.

\begin{tabular}{lcc}
\hline \multicolumn{1}{c}{ PROGENIE } & $\begin{array}{c}\text { "El Diamante" } \\
(950 \mathrm{msnm})\end{array}$ & $\begin{array}{c}\text { "San José" } \\
(1330 \mathrm{msnm})\end{array}$ \\
\hline Atzimba x R128.6 & 7.2 & 11.8 \\
Atzimba x DTO.28 & 7.3 & 9.4 \\
Atzimba x DTO.33 & 7.5 & 8.9 \\
Atzimba x 7XY.1 & 7.6 & 10.4 \\
Serrana x DTO.28 & 8.0 & 8.9 \\
Serrana x DTO.33 & 8.8 & 7.7 \\
\hline Duncan (p=0.05) & $\mathbf{1 . 4}$ & $\mathbf{2 . 7}$ \\
\hline
\end{tabular}


Tabla 6. Vigor y rendimiento de progenies de semilla híbrida y de polinización libre (PL) producidas en la Estación Experimental "San José" (1330 msnm). Evaluación realizada en "El Diamante" (950 msnm).

\begin{tabular}{lccc}
\hline & CoV* & $\begin{array}{c}\text { Sobrevivencia } \\
(*)\end{array}$ & $\begin{array}{c}\text { Rendimiento } \\
(\mathrm{kg} / \mathrm{m} 2)\end{array}$ \\
\hline 7XY.1 (PL) & 13.0 & 94.5 & 8.1 \\
DTO-28 (PL) & 12.2 & 95.7 & 6.4 \\
DTO-33 (PL) & 12.1 & 87.3 & 5.7 \\
R128.6 (PL) & 9.0 & 77.0 & 6.0 \\
Atzimba x R128.6 & 11.1 & 96.0 & -- \\
Serrana x 7XY.1 & 10.7 & 83.0 & -- \\
Serrana x R128.6 & 8.8 & 98.0 & $\mathbf{2 . 0}$ \\
\hline Duncan (p=0.05) & $\mathbf{2 . 0}$ & $\mathbf{9 . 3}$ &
\end{tabular}

* $\quad \mathrm{CoV}=$ Coeficiente de velocidad de emergencia

** $\quad$-- = No se obtuvieron los datos de cosecha.

\section{AGRADECIMIENTOS}

Sincero agradecimiento al organismo canadiense CUSO y a los Ings. Geoff Clare y Diana Paláez representantes de dicho organismo, por su colaboración logística. Igualmente, al Sr. Mario Rayo por su apoyo técnico, al Dr. Noel Palláis por sus importantes sugerencias y a los Srs. Rodolfo García y Juan Montenegro, productores de la finca "El Diamante", San Ramón, Matagalpa por su valiosa ayuda.

\section{REFERENCIAS BIBLIOGRÁFICAS}

1. Accatino P; Malagamba, P. 1982. Potato Production from TPS. International Potato Center. Lima, Perú. 20p.

2. Almekinders, C; Wiersema, S. 1985. TPS Production. True Potato Seed (TPS) Letler. 6:1. 
3. Atlin, G. 1984. Farmer maintenance of TPS varieties. In: Innovative Methods for Propagating Potatoes. Report XXVIII Plan. Conf. International Potato Center; Lima, Perú, p.32-62.

4. $\quad$ Centro Internacional de la Papa (1986). Informe Anual del CIP 1985. Lima, Perú. 184 p.

5. $\quad$ Centro Internacional de la Papa (1987). Informe Anual del CIP 1986. Lima, Perú. 232 p.

6. Centro Internacional de la Papa (1989). Informe Anual del CIP 1988. Lima, Perú. 226 p.

7. Engels, C; Sadik, S.; Bedewy, R.A. 1984. True Potato Seed (TPS) Research in Egypt. In: Innovative Methods for Propagating Potatoes. Report XXVIII Plan. Conf. International Potato Center Lima, Perú. p. 237-251.

8. El Bedewy, R; Engels, C.; Schwenkel, J. 1987. Uso potencial de la Semilla para la Producción de Papa en Egipto. CIP Circular 15:4.

9. Fernández, J. 1987. La Semilla sexual como una alternativa a la producción de papa en México. En: Memoria XIII Reunión Asociación Latinoamericana de la Papa (ALAP). Panamá, Panamá, p. 96-101.

10. Kidane-Mariam, H.M.; Mendoza, H.; Wissar, R. 1985. Performances of True Potato seed families derived from intermating tetraploid parental lines. Am. Potato J. 62:643-652.

11. Malagamba, P. 1982. Evaluation of Agronomic Technology for Potato Production from True Potato Seed. Technology Evaluation Series No. 1982-3. International Potato Center. Lima, Perú. 19 p.

12. Malagamba, P. 1983. Seed-bed substrates and nutrients requeriments for the production of the Potato seedlings. In: W.J. Hooker (ed). Research for Potato in the Year 2,000. Proc. Int. Cong. International Potato Center. Lima, Perú. 127-128.

13. Pallais, N.; Villagarcía, S.; Fong, N.; Tapia, J.; García, R. 1987. Effect of suplemental nitrogen on True Potato seed weight. Am. Potato J. 64: 482491.

14. Pallais, N.; Kalazich, J.; Santos-Rojas, J. 1986. The physical relationship between Potato berry and its seed. Hort. Science. 21: 1359-1360. 
15. Pallais, N. 1989. Investigación sobre la producción de semilla sexual de papa. Tercer Curso Internacional de Producción y Almacenamiento de Semillas de Papa. INIA/CIP/PNUD, Osorno, Chile. Enero 8 a Febrero 4, 1989.

16. Rodríguez, D. 1987. Evaluación de tubérculos-semillas obtenidos en almácigos a partir de semilla sexual en fincas de agricultores en TrujilloVenezuela. En: Memoria XIII Reunión Asociación Latinoamericana de la Papa (ALAP). Panamá, Panamá, p. 27-39.

17. Song, B.F.; Qu Don Yu; Vander Zaag, P. 1987. True Potato Seed in China: Past, Present and Future. Am. Potato J. 64: 321.

18. Upadhya, M. 1983. Studies on Potato Reproductive Biology for TPS Research in the year 2000. In; W.J. Hooker (ed.) Research for the Potato in the Year 2000. Proc. Int. Cong. International Potato Center. Lima, Perú. p. 180-181.

19. Upadhya, M; Thahur, K.C.; Juneja, A.; Kadian, M.S.. 1984. True Potato Seed Production; flowering, quality and economics. En: Innovative Methods for Propagating Potatoes. Report XXVIII Plan. Conf. International Potato Center. Lima, Perú. p. 117-147.

20. Wiersema, S. 1983. Potato Seed-Tuber Production from True Seed. En: WJ. Hooker (ed.) Research for the Potato in the Year 2000. Proc. Int. Cong. International Potato Center. Lima, Perú. p. 186-188.

21. Wiersema, S. 1984. The production and utilization of seed-tubers derived from True Potato seed. Ph.D., Thesis, University of Reading, England. $229 \mathrm{p}$.

22. Wiersema, S. 1986. A method of producing seed tubers from True potato seed. Potato Research. 29: 225-237.

23. Wissar, R.; Alvarado, J.; Rodríguez, D. 1987. Factibilidad del uso de semilla sexual en la producción comercial de papa en la región de los Andes de Venezuela. En: Memoria XIII Reunión Asociación latinoamericana de la Papa (ALAP). Memoria. Panamá, Panamá, p. 2739. 